\title{
Multilinguales
}

15 | 2021

Recherches algériennes en sociolinguistique et en littérature : discours scientifique/discours militant, quelles frontières?

\section{La lecture/déchiffrement en Algérie et la méthode syllabique}

Reading/deciphering in Algeria : proposal of the syllabic method

\section{Afaf Salhi et Mourad Bektache}

\section{OpenEdition}

Journals

Édition électronique

URL : https://journals.openedition.org/multilinguales/6266

DOI : $10.4000 /$ multilinguales.6266

ISSN : 2335-1853

Éditeur

Université Abderrahmane Mira - Bejaia

Référence électronique

Afaf Salhi et Mourad Bektache, «La lecture/déchiffrement en Algérie et la méthode syllabique »,

Multilinguales [En ligne], 15 | 2021, mis en ligne le 15 juin 2021, consulté le 14 février 2022. URL : http:// journals.openedition.org/multilinguales/6266; DOI : https://doi.org/10.4000/multilinguales.6266

Ce document a été généré automatiquement le 14 février 2022.

\section{c) $(1) \odot$}

Multilinguales est mise à disposition selon les termes de la Licence Creative Commons Attribution Pas d'Utilisation Commerciale - Pas de Modification 4.0 International 


\title{
La lecture/déchiffrement en Algérie et la méthode syllabique
}

Reading/deciphering in Algeria : proposal of the syllabic method

\author{
Afaf Salhi et Mourad Bektache
}

1 La lecture au sens du déchiffrage est une activité clé qui nécessite sans doute une maitrise totale de la part de l'apprenant. Pour cela, son enseignement constitue l'une des premières priorités à l'école primaire.

«La lecture est la première des trois connaissances de base dont l'école primaire

doit munir les élèves » (Giollito, $1984: 8$ ).

Au-delà de son usage fréquent au quotidien, la maîtrise de la lecture est une condition fondamentale de l'intégration socio- professionnelle de toute personne.

«Il faut bien admettre que la lecture conditionne la réussite scolaire au même

temps que l'individu » écrit Philibert (2006:14).

2 En fonction de leurs principes didactiques, les méthodes d'enseignement de cette activité sont élaborées essentiellement selon deux conceptions différentes: l'une, synthétique, basée sur le principe alphabétique c'est-à-dire aller de l'élément simple qu'est la lettre vers le texte en passant par le mot. L'autre, analytique qui va du texte vers la lettre. Elles visent chacune l'efficacité. Malgré les nombreux travaux de recherche qui ont traité de ce sujet, la question de rentabilité reste " relative » ouvrant ainsi le débat entre chercheurs et praticiens particulièrement les enseignants du cycle primaire. Ceux-ci leur revient de penser minutieusement au choix des méthodes adéquates du moment que les apprentissages ultérieurs dépendent de la maitrise de cette activité pendant cette étape de la scolarité.

3 En Algérie, l'apprentissage de la lecture débute en troisième année primaire (première année d'apprentissage du français langue étrangère) et se fait selon la méthode semi globale. Celle-ci étant une combinaison des deux méthodes syllabique et globale. Les instructions officielles de 2003 et celles de 2016 justifient le choix de cette méthode par le fait que son but est d'

«arriver à une lecture qui ne soit pas mécanique, qui saisisse les unités de sens en

s'appuyant sur chacune des articulations: lettre, syllabe, mot» (Document 
d'accompagnement décembre 2003: 6) (document d'accompagnement du programme $216: 7$ ).

Contrairement à cela, la méthode syllabique est, selon les mêmes documents,

«basée sur la présentation de phonèmes dépourvus de signification et vides de sens telle la syllabe » (Ibid. : p. 25).

4 Une première étude menée sur ce thème dans le cadre de notre mémoire de magister, nous a permis de relever un échec quant à l'appropriation de cette activité chez nos apprenants de troisième année primaire. Les résultats avaient révélé en effet, un taux de déficit considérable estimé à $66 \%$ de difficultés au niveau de la maitrise phonie/ graphie. Au niveau du déchiffrage, l'évaluation des apprenants a montré qu'il se limite nettement à une bonne prononciation des lettres suivant les étapes d'une leçon de lecture présentée selon la méthode semi-globale. Nous lions cet échec d'un côté, à la complexité de l'activité elle-même, la méthode semi-globale adoptée en plus de son apprentissage dans le cadre d'une langue étrangère.

5 Notre intérêt s'est suite à cela, retourné vers la proposition pour des apprenants algériens, d'une autre méthode d'enseignement qui pourrait les aider à maitriser cette activité et faire d'eux de bons lecteurs déchiffreurs, autonomes. Une méthode qui serait convergente avec les conditions d'apprentissage dont entre autres le milieu socioculturel et les capacités cognitives. La question fondamentale que nous soulevons est donc la suivante: serait-il plus efficace et plus bénéfique d'enseigner le déchiffrement selon la méthode syllabique à des élèves de troisième année primaire? Une question fondamentale de laquelle découlent d'autres : cette méthode sera-t-elle la bonne solution aux préoccupations des enseignants qui se plaignent du niveau de déchiffrage faible malgré les efforts fournis? Est-ce qu'un élève de troisième année primaire qui apprend selon la méthode synthétique peut être autonome dans son apprentissage et plus tard dans ses lectures?

\section{Cadrage théorique}

\section{Qu'est-ce que lire?}

6 L'acte de lire est défini selon plusieurs paramètres. C'est d'abord attribuer du sens aux signes graphiques en ce sens que l'on lie chaque lettre et /ou groupe de lettres à une sonorité particulière. «Lire » serait également en lien avec les capacités intellectuelles selon Muckensturm (2012:30). Ce dernier écrit que "c'est un travail de l'esprit " par lequel le lecteur parvient à "solliciter modestement mais réellement des facultés intellectuelles, la capacité d'invention »(P.8). Après quoi, l'auteur évoque la dimension $\mathrm{du}$ sens dans sa définition en écrivant que lire c'est: " créer du sens à l'aide des images visuelles que sont les mots» (Ibid.). Conjuguant les deux aspects décodage et compréhension, Chauveau (2004) enrichit cette idée en disant qu'il conçoit la lecture comme étant une "activité à la fois culturelle et langagière ». Elle est selon lui culturelle parce qu'on lit pour "s'informer, se divertir, agir, imaginer, apprendre... » .

7 Pour définir l'acte de lire, G. Chauveau et E. Chauveau (1990) rappellent des modèles théoriques dont essentiellement les modèles "de bas en haut " qui reposent sur une logique de passage des «éléments primaires» dont acquisition des lettres et syllabes vers des «processus cognitifs supérieurs » en relation avec la production du sens. Les modèles de « haut en haut » qui proposent une démarche contraire qui repose sur la 
reconnaissance des mots avec "anticipation des formes écrites" (Ibid). Bokstoel (2007) quant à elle, fait intervenir les indices syntaxiques, sémantiques et graphiques. Dans son travail, elle évoque la réflexion de Giasson qui pense que les connaissances sur la grammaire permettent à l'apprenant de faire la différence entre les mots qui se ressemblent. En effet, l'enfant arrive à lire convenablement des mots qui ont la même terminaison dès qu'il comprend qu'il s'agit d'un verbe dont le sujet est au pluriel, un nom ou un adverbe par exemple. L'enfant qui s'appuie sur le sens de la phrase arrive de ce fait à lire d'une façon automatique et spontanée les mots qui la composent. Enfin, concernant l'implication des signes graphiques dans la maitrise de la lecture, Bokstoel exige en plus de la connaissance des graphèmes, la maitrise de la forme des lettres script et cursive surtout pour les lettres dont la forme change complètement.

\section{Qu'est-ce qu'apprendre à lire ?}

8 C'est l'expérience qui sert à "recenser les savoir-faire » essentiels que fournit la maitrise du code et dont la finalité serait de réussir le décodage, explique Muckensturm (2012: 30). D'après le même auteur, apprendre à lire c'est passer par les niveaux de la lecture " hiérarchiques» dont la maitrise de l'un dépend de la maitrise de l'autre et qui sont: déchiffrage, lecture courante à haute voix, lecture silencieuse et enfin, lecture rapide (Ibid. : 30). De son côté, Giasson (2014) propose un canevas pour «l'évolution du jeune lecteur». Il s'agit d'une somme de capacités qui commencent par l'habileté de déchiffrement à travers la maitrise du code graphique aussi bien à l'oral qu' à l'écrit jusqu'à la capacité de choisir ses propres lectures en passant par plusieurs attitudes qu'un lecteur développe par rapport à un écrit dont l'anticipation, l'élaboration et l'exploitation qui ne sont autre que des aspects de la compréhension.

9 Afin de réaliser l'acte de lire, le processus commence par "une exploitation visuelle" (Gombert, 2014) qui consiste en une fixation d'une chaine de mots au même temps, suivie d'une identification des mots écrits (Ibid.). Celle-ci serait le résultat d'une compréhension du principe alphabétique se basant sur la correspondance graphie/ phonie. Après quoi, le lecteur entame l'identification des graphèmes dont lettres et groupes de lettres (l, $r, \mathrm{ch}$, in, eau...) suivie par un apprentissage explicite de ces derniers. C'est une acquisition importante au début de l'enseignement car elle prépare à l'auto-apprentissage (Ibid.). Le principe alphabétique constitue également une étape importante dans l'apprentissage de la lecture selon Gombert(2014). L'apprenant ne doit pas se contenter d'identifier les lettres mais il doit comprendre qu'elles entrent dans la formation d'autres mots (Ibid.). Ces acquisitions constituent un dispositif indispensable au déchiffrement. Ce dernier étant «l'étape clé de la lecture » (Dehaen, 2007 :291).

\section{Spécificité de la lecture en français}

Des études telles que celle de Peerman et al. (2013) ont montré que la spécificité de la lecture en langue française réside dans "la consistance» des correspondances graphèmes / phonèmes du moment qu'à plusieurs graphèmes correspond un phonème. Les apprenants doivent de ce fait, adopter une démarche phonologique en début d'apprentissage de la lecture car la réussite dans l'appropriation de l'habileté de lire en dépendrait. Les études de Charolles menées entre 2003 et 2016 ont prouvé que des apprentis lecteurs favorisent la voie phonologique pour lire aussi bien des mots réguliers que des mots irréguliers même si des erreurs de régularisation sont à 
mentionner. Les enfants ont tendance en effet, à prononcer des lettres muettes comme le $\mathbf{p}$ de sept.

Malgré ces insuffisances recensées, les recherches de Sprenger Charolles ont confirmé que les apprenants qui ont le plus recours au décodage c'est-à-dire qui maitrisent l'assemblage et la prononciation correcte des syllabes sont ceux qui progressent le plus dans l'apprentissage de la lecture/déchiffrement. En effet, le choix de cette stratégie favorise des connexions entre graphèmes et phonèmes.

\section{Apprendre à lire en langue étrangère}

11 Gaonach (2005), affirme que des mécanismes en interaction qui entreraient dans la réalisation de l'acte de lire, seraient déjà placés en langue maternelle. De ce fait, la lecture dans une autre langue n'aurait pas un début (ibid.). Cet appui sur la langue maternelle afin de lire dans une autre langue, se réaliserait de la même façon qu'il soit sous la forme d'une prise d'informations à partir d'hypothèses ou une prise d'informations à partir de lettres ou de mots (Goodman et Slith in Gaonac'h : 2005). Un avis qui n'est pas partagé par Riquois (2010) qui pense qu'il s'agit en fait de «deux situations de lecture différentes» dont chacune présente "des particularités et des objectifs singuliers» (Ibid). La différence réside également au niveau du comportement adopté par l'apprenant vis-à-vis le texte. Ainsi, un apprenant en français langue étrangère ou seconde, est considéré comme un "sujet lecteur» dans sa langue maternelle et « lecteur apprenant » quand il lit un texte en français.

Lors de l'appropriation du système de l'écrit durant la phase préparatoire, les apprenants aussi bien natifs que non francophones rencontrent des difficultés qui entravent leur apprentissage à cette étape (Rafoni : 2014). Les principales contraintes que rencontrent des apprenants non francophones se situent essentiellement au niveau de la distinction des unités non significatives qu'ils ont tendance à acquérir un peu tard par rapport aux natifs. D'autre part, l'identification du mot écrit à partir des lettres et des syllabes écrites est entravée à cause du stock lexical limité chez les enfants non francophones. Le dernier point soulevé par Rafoni quant aux difficultés rencontrées par les apprenants dont le français n'est pas une langue maternelle, concerne «les différences » dans le "rythme d'acquisition (Ibid.). Ce problème s'explique selon l'auteur par un déficit dans la manipulation des unités linguistiques par les allophones dépassés en cela largement par leurs pairs francophones (Ibid.).

\section{Débat des méthodes d'enseignement de la lecture}

De par leurs principes méthodologiques, les méthodes d'enseignement de la lecture se divisent en dépit de plusieurs désignations (phonétiques, alphabétiques, globales, semi globales) en deux grands ensembles: les méthodes analytiques qui favorisent la décomposition d'un tout dont un texte ou une phrase pour aller vers la syllabe puis la lettre. D'autres dites synthétiques qui, au contraire, s'appuient sur la composition à partir de la plus petite unité (la lettre) pour aller vers des éléments plus complexes dont la syllabe, le mot, la phrase et enfin le texte.

Pour enseigner la lecture au sens du déchiffrement, deux grands axes font l'unanimité, le décodage des signes graphiques et l'accès au sens. Cependant, un grand débat qui retourne parfois en «querelle» fait que les chercheurs polémiquent sur le 
cheminement qu'il faut adopter pour y parvenir. Condamnée à l'échec par les chercheurs, la méthode dite "idéo visuelle » est la seule qui semble "bonne à écarter " car, à en croire Boulanger (2010:143), elle :

refuse le travail systématique sur la correspondance graphème/phonème. Elle n'est plus utilisée et personne ne pense sérieusement aujourd'hui qu'un enfant puisse apprendre de textes (...).

Un point de vue partagé par Peiron (2015) qui, lui aussi, appelle à délaisser cette méthode car elle " consiste à photographier les mots, à les reconnaitre grâce à leur forme ".

Une étude américaine menée par l'organisme de recherche "national Reading panel » entre 1988 et 1999 a conclu également que les méthodes purement globales qui excluent complètement l'enseignement des correspondances graphies/phonies, sont démunies de la moindre efficacité. Elles reposent sur la mémorisation de la graphie des mots sinon sur la reconnaissance des mots à travers le sens de la phrase. D'autant plus que cette stratégie qui conviendrait plus aux écritures idéographiques ne peut convenir à une langue alphabétique.

Dans une conception différente, Charmeux (2016) écarte toute entrée par la correspondance graphème/phonème la considérant comme une "erreur pédagogique " qui risque de mettre en difficulté tous les élèves. Elle explique son point de vue comme suit : les enfants entrent à l'école avec des représentations graphiques sur les objets qu'ils possèdent et qu'ils voient. Il serait donc préférable de leur enseigner la lecture à partir de ce qu'ils savent. Aborder l'apprentissage de la lecture à partir d'éléments abstraits, veut dire selon Charmeux, « casser dès l'entrée » pour l'élève " toute possibilité de comprendre »et prévoir des répercussions négatives sur l'habileté de lire (Ibid.).

\section{L'évaluation scientifique des méthodes d'enseignement de la lecture}

Devant ce débat « acharné » sur les différentes méthodes d'enseignement de la lecture, la neuroscience intervient afin de fournir un avis aussi bien objectif que rigoureux. Ainsi, Stanislas Dehaen affirme que

«les données de l'imagerie et de la psychologie ne sont pas neutres vis-à-vis des grands débats sur la lecture à l'école » (2007:290).

Afin de justifier le recours à l'imagerie médicale dans le domaine, il affirme que « quand il faut apprendre à lire, tous les enfants ont le même cerveau qui impose les mêmes contraintes et la même séquence d'apprentissage » (Ibid.).

Cet avis serait de ce fait, suffisant pour retracer les différentes étapes par lesquelles passe le parcours de déchiffrage des mots

«notre cerveau ne passe pas directement de l'image des mots à leur sens. A notre insu, toute une série d'opérations cérébrales et mentales s'enchainent avant qu'un mot ne soit décodé » (Ibid.).

Pour justifier le choix de la méthode synthétique, il affirme que

«l'imagerie médicale met en évidence l'effet le plus spectaculaire : l'hémisphère droit s'active pour la lecture globale, alors que l'attention portée aux lettres active la région classique de la lecture l'aire occipito-temporale ventrale gauche. Autrement dit, l'apprentissage par la méthode globale mobilise un circuit inapproprié » (Ibid.).

Une idée soutenue par Thérèse Cuche et Michelle Sommer (2016) qui, s'appuyant sur leur expérience d'orthophoniste et psychothérapeute, confirment qu'il faut apprendre à l'enfant le code pour qu'il devienne lecteur déchiffreur. Lui apprendre un texte par 
cœur, lui donne juste l'impression qu'il lit (Ibid.).Pire encore, opter pour une démarche globale mènerait une catégorie d'apprenants à l'abandon de cet apprentissage (Ibid.).

Les adeptes de cet avis n'ont pas négligé de proposer quelques recommandations didactiques pour la préparation et la présentation d'une leçon de lecture selon la méthode syllabique dont :

- Préparer l'enfant à travers l'entrainement à la conscience phonétique en focalisant sur une bonne perception de la forme des lettres

-Aborder la correspondance graphème/phonème au début du processus enseignement/ apprentissage d'une façon explicite

- Procéder dans l'enseignement des lettres selon les critères du degré de complexité et de la fréquence

- Expliquer qu'à chaque son correspondent plusieurs transcriptions

- Proposer aux apprenants des mots et des phrases qui contiennent uniquement les lettres étudiées.

\section{Choix méthodologique}

Pour mener notre étude, nous avons proposé une progression conçue selon les principes de la méthode syllabique. Nous l'appliquons dans des classes de troisième année primaire qui constitue la première année d'apprentissage du français langue étrangère en Algérie. Grâce aux neuf évaluations programmées le long du processus enseignement/apprentissage qui dure une année scolaire, nous aurons des indices sur l'apport des principes méthodologiques de cette démarche par rapport à l'acquisition de l'acte de lire pour des apprentis -lecteurs. Nous serons également renseignés sur l'adéquation de cette méthode avec les conditions d'apprentissage de cette activité.

\section{Conditions du déroulement de l'enquête}

21 L'étude est menée dans dix écoles de la circonscription f2 de la wilaya de Constantine. L'échantillon comporte 377 élèves répartis sur 15 classes. Le choix dans sa délimitation est basé sur les paramètres du milieu social, le nombre d'élèves dans chaque classe et l'expérience professionnelle des enseignants relative au nombre d'années de service. Notre choix n'a pas pu être complètement satisfait à cause de quelques obstacles liés essentiellement à la réticence de certains enseignants qui ont refusé de participer à l'étude sinon le départ de certaines d'entre elles travaillant dans des régions qui répondent à nos critères, en congés. Nous avons quand même réussi à diversifier notre choix qui s'est fixé sur les écoles suivantes:

\section{Dans la région urbaine}

- L'école Aboubakr Essedik dans la région Djebel Alouahche

- L'école Mouloud Feraoun dans la cité Emir Abdelkader

- L'école Loucif Fatima dans la cité Loucif au centre-ville.

\section{Dans la région semi-urbaine}

- L'école Goumida Ali à 45 kilomètres de Constantine

- L'école Hanache Rabah dans la cité Sarkina dans la banlieue 
- L'école Boughrara Rabah à 45 Kilomètres de Constantine

\section{Dans la région rurale}

- L'école Houcine Chaaraoui dans la région Tafrente

-L'école Seradj Mostapha dans la zone rurale de Djebel Aouahch

\section{Dans la région semi-rurale}

- L'école Aouane Rabah à 45 kilomètres de Constantine.

\section{L'instrumentation}

Nous avons procédé avec une progression de notre conception qui contient le programme à enseigner en plus d'une fiche d'évaluation qui sert à la quantification du rendement des apprenants concernant la qualité et le rythme de l'appropriation de l'habileté de lire durant une année scolaire. Nous avons conçu la progression dans une logique qui

«(...) suppose avant tout tenir compte des besoins et des souhaits de la classe »

(Courtillon, $2003: 10$ ).

Nous la présentons en deux volets. A travers le premier, nous décrivons le principe synthétique de la méthode qui consiste en un départ de l'élément le plus petit qu'est la lettre. La distinction voyelle /consonne nous mène vers le montage syllabique qui entre dans la construction du mot, puis la phrase et enfin le texte.

Concernant la compétence qu'on tend à installer chez l'apprenant à la fin de son parcours d'apprentissage, c'est le rendre capable d'effectuer une lecture spontanée dans toutes les situations de l'apprentissage et qu'il soit surtout autonome dans ses lectures. Au terme de chaque séance d'enseignement/apprentissage de la lecture, nous aspirons à ce que l'apprenant arrive à identifier le ou les graphèmes sujet (s) d'étude sur le plan audio-visuel, et qu'il arrive à comprendre la logique d'assemblage (montage syllabique) du plus simple vers le plus complexe (consonne/voyelle- consonne/semivoyelle, consonne/sons plus complexes : voyelles nasales par exemple).

Dans le même volet, nous avons inclus la démarche qu'un enseignant adopte dans la présentation d'une leçon de lecture/déchiffrement selon la méthode syllabique. Ainsi, le cheminement rejoint les principes de la méthode en ce sens que l'enseignant commence par une présentation du ou des graphèmes à étudier, après quoi, il passe au montage syllabique suivi d'un montage d'une série de mots avec la participation des apprenants. Afin de s'assurer que ces derniers ont compris la logique de leur apprentissage et afin de vérifier l'atteinte des objectifs délimités au préalable, l'enseignant leur demande de lire les mots proposés. La constitution progressive d'un stock lexical, permet le passage à la rédaction par l'enseignant de petites phrases et plus loin de petits paragraphes à partir de mots qui contiennent uniquement les lettres étudiées.

Dans ce volet, nous avons également intégré les directives et les consignes destinées aux enseignants pour les aider dans leur pratique de classe. En effet, même si nous tenons au respect strict des principes méthodologiques de la méthode dont le passage progressif des éléments simples vers ceux plus complexes, nous optons pour une autonomie de l'enseignant concernant la modification des étapes d'une leçon en fonction du rythme d'apprentissage par exemple. Il revient également au praticien de 
travailler à partir des exemples de la progression proposée, les enrichir ou de choisir d'autres. Nous avons également recommandé le traitement des difficultés relatives au système graphique de la langue française dont présence de lettres muettes, la liaison ainsi que les exceptions telles le « er » qui se prononce parfois [e] et parfois [ère]. Quant au paramètre compréhension, nous avons rappelé la nécessité de lui réserver tout l'intérêt en pensant à des stratégies et des moyens qui le facilitent dont illustrations, objets concrets, dessins et dramatisation des scènes dans la mesure du possible.

Nous exposons et décrivons à travers le deuxième volet, les différents paramètres de la progression proposée. Nous nous sommes efforcée à établir un enchainement « rationnel » qui tend à un maximum d'efficacité et de rentabilité. Ainsi, il est question dans un premier temps, d'initier les apprenants à l'apprentissage de la lecture / déchiffrement en commençant par une mémorisation des lettres de l'alphabet afin de fournir une entrée "fluide » dans le processus d'acquisition de la lecture et préparer à des apprentissages plus complexes. Il est également question durant cette phase, de décrire le système vocalique français en faisant d'abord la distinction voyelle/consonne ensuite en expliquant la logique du montage syllabique. En effet, l'apprenant novice doit comprendre à ce stade, que c'est la voyelle qui donne la résonnance à la consonne. Dans le même volet, nous présentons la progression des apprentissages en commençant par l'étude des voyelles suivie par celle des semi-voyelles ou et oi. Cet apprentissage st justifié à ce stade par l'identification auparavant des différentes voyelles ce qui permet ce type de combinaisons. D'autre part, les semi-voyelles permettront le montage d'un maximum de syllabes ce qui donnera une diversité de mots isolés et de phrases et à lire.

Après acquisition des voyelles et des semi-voyelles ou et oi, nous passons à l'étude des consonnes. Celle-ci est une phase complexe durant laquelle l'apprenant reçoit deux types d'apprentissage : il continue l'identification des lettres et entame la combinaison et la production des mots et des phrases. Nous terminons par l'étude des sons. Il s'agit de combinaisons de graphèmes qui produisent des sonorités: digrammes ${ }^{1}$ et/ou trigrammes ${ }^{2}$. Nous les proposons après l'étude de toutes les lettres pour que l'apprenant progresse dans le processus d'apprentissage en enrichissant les combinaisons allant du plus simple vers le plus complexe (passer de syllabes de deux lettres à des syllabes de trois lettres et plus). Cela lui permet d'aborder le déchiffrement de paragraphes de plus en plus longs.

Pour élaborer le programme à enseigner, nous nous sommes appuyée sur les principes de jumelage de certaines lettres pour une meilleure opposition phonologique telles : $\mathbf{m} / \mathbf{n}-\mathbf{t} / \mathbf{d}-\mathbf{f} / \mathbf{v}$. Nous avons procédé également selon la ressemblance grapho/ phonétique pour jumeler certains sons. C'est le cas des transcriptions des sons [o], [e], [ع],[ $\varepsilon$ :] [ə], [oe],[ø],des voyelles nasales an/am- on/om - en/em- un/um - in/ain / ein)ainsi quedes sons ail/aille- eil/eille- euil/euille- ouil/ouille. Enfin des suites de voyelles et voyelles/consonnes : (ia/io/iel -ier/iére- ien/iènne). Par rapport à leur singularité phonétique, nous avons proposé l'étude de certaines lettres séparément. C'est le cas du $\mathbf{l}, \mathbf{j}$ et $\mathbf{r}$. Nous basant sur le même critère, nous avons procédé de la même façon avec certains sons.

Enfin, concernant la progression des apprentissages, nous avons procédé essentiellement selon les critères du taux de fréquence des lettres et des sons dans la langue, par rapport au nombre de mots qui contiennent telle lettre et qu'un enfant algérien peut comprendre en français. Enfin, par rapport à la dépendance des sons les uns par rapport aux autres dans la constitution des mots lisibles. La fiche d'évaluation 
ou fiche de suivi réservée au contrôle de l'évolution de l'apprentissage contient les éléments suivants : le numéro de l'épreuve, son contenu, un tableau avec une première case réservée à la liste nominative des apprenants et une autre qui informe sur le niveau de déchiffrement. Celle-ci est subdivisée en colonnes dont chacune fournit des informations de plus en plus affinées sur le niveau de lecture, ensuite sur la nature des lacunes décelées chez l'apprenant. Nous y trouvons les indices suivants: niveau de lecture très bon (TB)- niveau bon (B) - moyen avec déficit au niveau de l'acquisition de la lettre (M.A.L)- moyen avec déficit au niveau de la combinaison (M.A.C)- très faible au niveau de l'acquisition de la lettre (T.F.A.L) - très faible au niveau de l'acquisition de la combinaison (T.F.A.C).

\section{Les modalités d'évaluation}

Pour la première épreuve, nous avons opté pour une dictée afin de contrôler l'acquisition des lettres et des syllabes, éléments indispensables à l'apprentissage de la lecture. Nous estimons que contrairement à la distinction auditive, la reconnaissance visuelle de ces petites unités n'est pas suffisante pour le contrôle de leur acquisition. A partir de la deuxième épreuve, nous optons pour des épreuves de lecture car l'apprenant aurait développé à ce stade de l'apprentissage une conscience phonologique (discrimination des différents phonèmes d'un mot) qui lui permettrait de découvrir l'unité mot aussi bien oralement qu'à l'écrit. Plus l'apprenant progresse dans son apprentissage, on lui propose des listes plus élaborées de mots ainsi que quelques phrases et/ ou des paragraphes.

\section{Présentation et interprétation des résultats}

31 Pour interpréter les résultats, nous procédons à une catégorisation des épreuves selon les étapes du processus enseignement/apprentissage. Ainsi, nous divisons ce procédé en trois étapes : celle de l'acquisition des lettres régulières et des syllabes simples, elle est constituée des quatre premières épreuves, ensuite, l'étape de l'appropriation des lettres à irrégularité phonétique qui ont une double voire une triple prononciation. Celle-ci englobe les deux épreuves cinq et six. La troisième étape est celle de l'acquisition des sons complexes. Elle est constituée des épreuves sept, huit et neuf.

Afin de pouvoir cerner les différents éléments qui concernent l'objectif de l'étude, nous prenons en considération les paramètres suivants : le contenu des épreuves, le nombre d'apprenants par classe, l'expérience des enseignants de l'échantillon relative au nombre d'années de service et enfin l'emplacement de chaque école. Les scores que nous présentons représentent une moyenne des scores de chaque étape pour chaque indice.

\section{Première étape}

Fig1 : la qualité de rendement des apprenants durant la première étape de l'enseignement/ apprentissage

$$
\text { Indices Moyenne du score réalisé }
$$




\begin{tabular}{|l|l|}
\hline B & 26,43 \\
TB & 23,09 \\
T.F.A.L & 16,92 \\
M.A.C & 14,59 \\
M.A.L & 13,11 \\
T.F.A.C & 08,16 \\
\hline
\end{tabular}

Commentaire: Précisons d'abord que nous classons les résultats du rendement des apprenants en deux catégories: celle des acquis ou la réussite représentée par les indices «très bon » et bon »; celle des « déficits » représentée par les indices : « moyen au niveau de l'acquisition de la lettre ", « moyen au niveau de la combinaison », « très faible au niveau de l'acquisition de la lettre ", "très faible au niveau de la combinaison ». Les résultats des premières épreuves montrent clairement un niveau de rendement positif à cette étape de l'apprentissage du moment que les deux indices «bon" et «très bon " s'affichent clairement à travers des pourcentages importants avec une prédominance de l'indice "bon». Les apprenants ont en effet, "pu surmonter " beaucoup de difficultés pendant les premières épreuves de déchiffrage en ce sens qu'ils ont pu identifier les lettres étudiées à cette étape, effectuer convenablement des combinaisons et décoder de ce fait les mots qui leurs sont proposés.

Notons également que les meilleurs résultats pour le niveau "bon ", sont enregistrés dans les classes où le nombre d'apprenants atteint une moyenne de 21 élèves et la moyenne de l'expérience professionnelle des enseignantes atteint une moyenne de 10 ans de service ; et ce, par rapport à l'ensemble des classes où la moyenne des élèves est estimée à 24 élèves et l'expérience professionnelle des enseignantes est estimée à 5 ans. Par rapport au milieu socio culturel, le niveau «bon » est mentionné avec différentes échelles dans les écoles de toutes les régions concernées par notre étude ; notamment dans les zones : urbaine, semi urbaine, rurale et semi rurale.

L'analyse des déficits durant cette étape de l'enseignement/ apprentissage, révèle que le taux de faiblesse le plus important est mentionné pour l'indice « très faible au niveau de l'acquisition de la lettre » suivi d'un niveau «moyen dans l'acquisition du système combinatoire ». La faiblesse dans l'acquisition de l'unité lettre se justifierait à cette étape de l'apprentissage par l'implication des apprenants dans l'appropriation d'une langue étrangère. Les lettres proposées à cette étape, sont au nombre de dix: six voyelles et quatre consonnes; alors que l'évaluation est programmée après à peu près trois semaines d'enseignement /apprentissage. Même si nous avons tenu à entamer l'enseignement avec des phonèmes qui sont «familiers » aux apprenants tels que [m], [n] et [d], les "images» proposées restent nouvelles et certaines d'entre elles pourraient entrainer des confusions visuelles.

36 Concernant le déficit dans l'appropriation du principe combinatoire, le résultat mentionné trouverait une explication par rapport aux contenus qui ont consisté au départ en un déchiffrement de lettres, de syllabes voire de mots avec des combinaisons simples constituées de syllabes du type consonne + voyelle. Durant la deuxième épreuve, ont été proposés des mots et des phrases avec des syllabes complexes à savoir, consonne + semi-voyelle. Le passage d'un niveau de lecture à un autre aurait entrainé des difficultés au niveau de l'assemblage des syllabes. Après deux épreuves qui ont 
proposé des contenus de niveaux différents, une diminution du taux de déficit est constatée au cours de la troisième épreuve. Cela témoignerait d'un passage vers la «maitrise » des contenus enseignés au niveau de l'identification ainsi qu'au niveau des mécanismes de déchiffrage.

Les taux les plus élevés de "faiblesse » dans l'acquisition de la lettre sont mentionnés pour l'ensemble des classes où la moyenne de l'effectif est égale à 24 élèves et la moyenne de l'expérience professionnelle des enseignantes est estimée à 6 ans d'exercice ; et ce, par rapport aux classes où la moyenne des élèves est estimée à 25 et la moyenne des années de service des enseignantes est estimée à 9 ans de service. Le niveau d'acquisition moyen dans l'appropriation du système combinatoire, est mentionné avec des taux élevés dans les classes où la moyenne de l'effectif atteint 26 élèves et l'expérience professionnelle des enseignantes atteint 8 ans de service ; par rapport à celles où la moyenne de l'effectif est estimée à 17 élèves et la moyenne de l'expérience professionnelle des enseignantes atteint 3 ans.

Un "déficit » important représenté par les deux indices «très faible au niveau de l'acquisition de la lettre» et "moyen au niveau de l'acquisition du système combinatoire", est mentionné dans les écoles des quatre régions: urbaine, semi urbaine, rurale et semi rurale. La prédominance est pour les écoles de la région semi urbaine qui ont connu le plus grand taux de carence.

Nous récapitulons à travers l'histogramme suivant, la qualité du niveau de rendement au cours de cette première étape de l'enseignement / apprentissage.

Fig2 : la qualité de rendement des apprenants durant la première étape de l'enseignement/ apprentissage

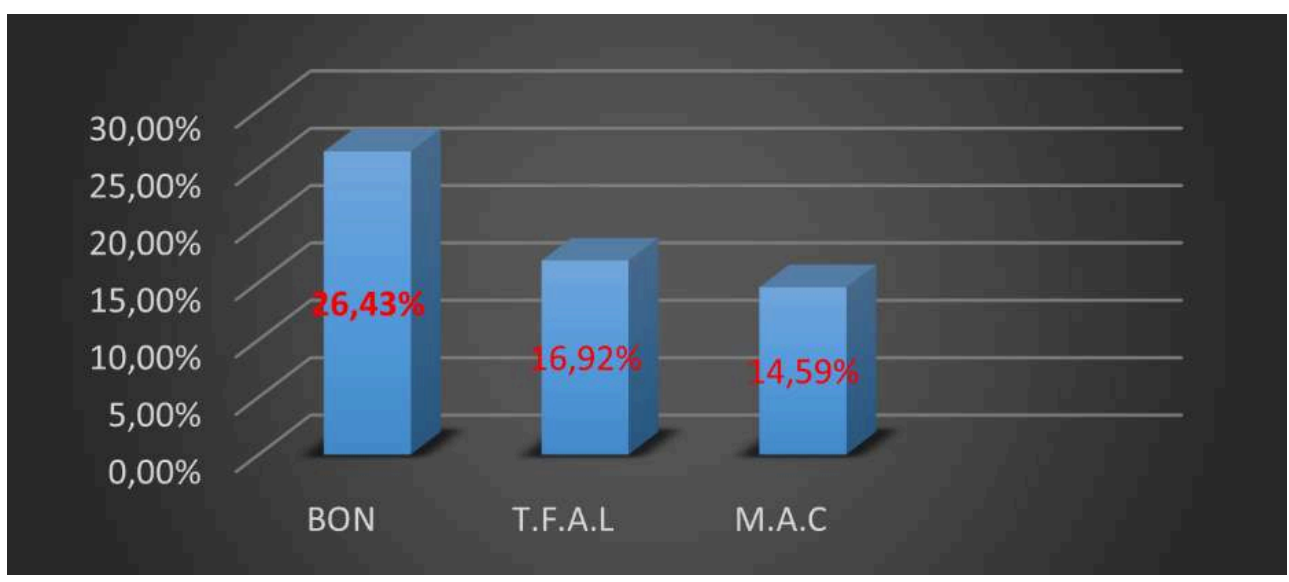

\section{Deuxième étape}

Fig3 : la qualité de rendement des apprenants durant la deuxième étape de l'enseignement/ apprentissage

Indices Moyenne du score réalisé 


\begin{tabular}{|l|l|}
\hline TB & $30,37 \%$ \\
B & $23,88 \%$ \\
M.A.C & $12,80 \%$ \\
T.F.A.L & $12,29 \%$ \\
M.A.L & $11,49 \%$ \\
T.F.A.C & $08,02 \%$ \\
\hline
\end{tabular}

Commentaire : Les résultats de cette deuxième étape d'enseignement/ apprentissage révèlent par rapport au paramètre "réussite » une nette amélioration du niveau de déchiffrement du moment que l'indice " très bon " s'affiche en tête des résultats avec le pourcentage le plus élevé. Au cours du deuxième trimestre, un nombre "important " d'apprenants a de ce fait, pu maitriser les éléments que nous jugeons « indispensables » au déchiffrement à savoir l'identification de l'unité lettre ainsi que le principe d'assemblage c'est-à-dire la combinaison des syllabes au niveau de la fusion : consonne/ voyelle puis l'assemblage des syllabes afin de prononcer les mots. La maitrise des premières unités et la lecture de mots au cours de la première étape semble "faciliter " le déchiffrement d'une unité plus complexe qui est la phrase. Signalons également, que cet indice a connu une hausse entre la cinquième et la sixième évaluation en passant de $29,73 \%$ à $31,01 \%$. Concernant le paramètre " déficit ", un score presque à égalité est enregistré pour les deux indices "moyen dans l'acquisition du principe combinatoire " suivi de celui « très faible dans l'acquisition de la lettre ».

Basée sur le principe d'assemblage, l'inculcation de ce principe pour un élève qui apprend à lire avec la méthode syllabique, revient pendant les séances d'apprentissage ; son contrôle se fait régulièrement à travers les différentes évaluations ce qui développe chez le lecteur débutant un " automatisme » qui l'aide à déchiffrer au fur et à mesure le long de sa formation. L'apprentissage des lettres par contre, confronte l'apprenant dans chaque séance d'apprentissage à la découverte de nouveaux signes qui différent les uns des autres de par leur forme et/ou leur correspondance phonétique. Au cours de cette deuxième étape réservée à l'apprentissage des lettres à irrégularité phonétique, le niveau de rendement «bas » enregistré pendant l'épreuve 6 est relatif à l'enseignement des lettres $\mathbf{x}$ et $\mathbf{y}$. Ces deux graphèmes n'ont pas de règles « précises » de prononciation. La lettre $\mathbf{x}$ est prononcée $\mathbf{k s}$ ou $\mathbf{g z}$ entre deux voyelles dans « exercice » et « taxi » et entre une voyelle et une consonne comme "mixte " par exemple. Il en est de même pour la lettre $\mathbf{y}$. Même si elle a la valeur de ii, celle-ci apparait comme une consonne dans les mots où elle précède une voyelle tels que " voyage " et " rayures " , et elle est exclusivement une voyelle qui produit [i] dans « stylo » et " pyjama » par exemple. En l'absence d'une "règle de prononciation ", l'apprenant se confronte à une situation de «doute » et de "tâtonnement » afin d'effectuer la bonne combinaison surtout qu'à ce stade, il ne dispose pas d'un stock lexical « riche » qui pourrait l'aider à déchiffrer en reconnaissant le mot.

41 Contrairement à cela, et même si les lettres $\mathbf{s}, \mathbf{c}$ et $\mathbf{g}$ (programmées avant $\mathbf{x}$ et $\mathbf{y}$ ), sont à irrégularité phonétique, leur valeur reste quand même systématique par rapport aux voyelles auxquelles elles sont associées. Ainsi, le $\mathbf{s}$ est toujours prononcé [z] entre deux voyelles, le $\mathbf{c}$ est toujours prononcé [k] devant $\mathbf{a}, \mathbf{o}, \mathbf{u}$ et prononcé [s] devant $\mathbf{i}, \mathbf{e}$, é. Le $\mathbf{g}$ est toujours doux [j]devant $\mathbf{i}, \mathbf{e}$, é et dur $[\mathbf{g}]$ devant $\mathbf{a}, \mathbf{o}$ et $\mathbf{u}$. Les meilleurs scores réalisés pour le niveau "très bon » dans la réalisation de l'acte de lire sont indiqués 
pour l'ensemble des classes, où la moyenne de l'effectif est égale à 19 élèves et la moyenne de l'expérience professionnelle des enseignantes est estimée à 10 ans de service; et ce, par rapport à l'ensemble des classes où la moyenne de l'effectif est estimée à 32 élèves et l'expérience professionnelle des enseignants est estimée à 4 ans de service.

Les résultats supérieurs à la moyenne pour l'indice "moyen au niveau de la combinaison » sont indiqués dans l'ensemble des classes où la moyenne de l'effectif est égale à 30 élèves et la moyenne de l'expérience professionnelle des enseignantes est égale à 8 ans de service et ce; par rapport aux classes où la moyenne de l'effectif est estimée à 25 élèves et l'expérience professionnelle des enseignantes est estimée à 3 ans de service. Concernant le troisième indice "très faible dans l'acquisition de la lettre ", un déficit important est mentionné dans l'ensemble des classes où la moyenne de l'effectif est égale à 28 élèves et la moyenne de l'expérience professionnelle des enseignantes est égale à 6 ans de service; et ce, par rapport à l'ensemble des classes où la moyenne de l'effectif est égale à 24 élèves et la moyenne de l'expérience professionnelle des enseignantes est égale à 6 ans de service.

Un niveau de réussite important (supérieur à la moyenne) concernant cette étape d'enseignement /apprentissage est mentionné à égalité dans les quatre zones : urbaine, rurale semi urbaine et semi rurale. Par ailleurs, un niveau inférieur à la moyenne est mentionné à égalité dans les trois régions: urbaine, semi urbaine et semi rurale. Concernant le paramètre " déficit ", des niveaux supérieurs à la moyenne sont signalés dans les quatre régions concernées par notre étude avec une prédominance dans la région semi urbaine. Des niveaux « moins importants » (inférieurs à la moyenne) sont indiqués également dans les quatre régions avec une prédominance des écoles de la région semi rurale.

Nous récapitulons à travers l'histogramme suivant, la qualité du niveau de rendement au cours de cette deuxième étape de l'enseignement/ apprentissage.

Fig4 : la qualité de rendement des apprenants durant la deuxième étape de l'enseignement/ apprentissage.

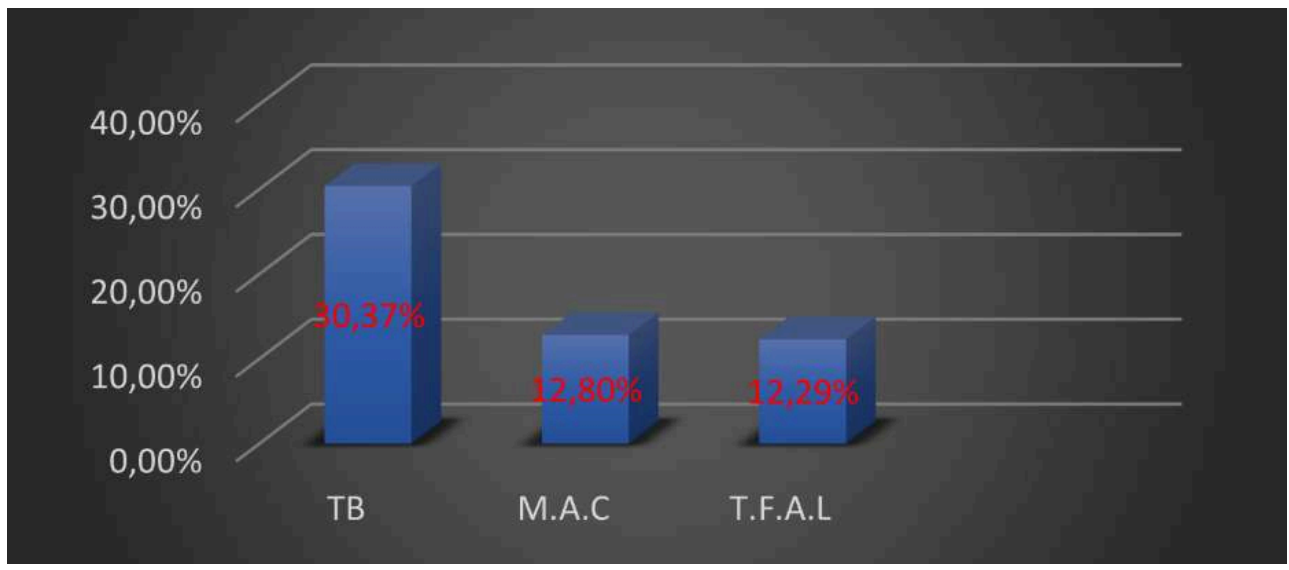




\section{Troisième étape}

A ce niveau, nous avons remplacé les abréviations ( M.A.L) et (T.F.A.L) par (M.A.S) et (T.F.A.S) c'est-à-dire moyen au niveau de l'acquisition des sons et très faible au niveau de l'acquisition des sons.

Fig5 : la qualité de rendement des apprenants durant la troisième étape de l'enseignement/ apprentissage

\begin{tabular}{|l|l|}
\hline Indices & Moyenne du score réalisé \\
\hline B & $29,45 \%$ \\
\hline TB & $21,30 \%$ \\
\hline M.A.C & $16,25 \%$ \\
\hline T.F.A.S & $15,83 \%$ \\
\hline M.A.S & $15,61 \%$ \\
\hline T.F.A.C & $07,04 \%$ \\
\hline
\end{tabular}

Commentaire : 1 est très important de noter que les classes ne sont pas toutes parvenues à atteindre cette étape et donc à terminer le processus enseignement/ apprentissage selon le programme que nous avons conçu. Les résultats révèlent tout de même, une confirmation de la prédominance du paramètre "réussite ", du moment que l'indice «bon » s'affiche à travers le plus haut pourcentage. Il est en écart « assez étroit » par rapport à l'indice " très bon » qui s'affiche en deuxième position. A la fin de ce parcours d'acquisition de l'habileté de lire à travers la méthode syllabique, les résultats de la troisième étape reflètent un parcours d'apprentissage "stable " par rapport aux résultats requis. Cette "stabilité » est maintenue malgré le principe de la graduation des difficultés adopté dans la conception des contenus proposés à ce niveau et qui ont consisté en des syllabes complexes constituées de quatre voire de cinq et de six lettres (travail, fenouil, conseille) ainsi que des passages sous forme de phrases et de paragraphes constitués de quatre, vingt, voire même de trente-six mots. Précisons que le paramètre "réussite » est représenté à travers l'indice "bon » durant cette dernière étape de l'enseignement/apprentissage et ce, par rapport à l'étape précédente où les résultats ont témoigné d'un niveau « très bon ».

Concernant le paramètre " déficit », le plus haut niveau de «carence » est signalé dans l'acquisition du principe d'assemblage à travers l'indice «moyen dans l'acquisition de la combinaison ».Cette « lacune » pourrait s'expliquer par la «double » complexité du principe d'assemblage à ce niveau du processus d'apprentissage sur le plan graphophonétique. L'apprenant s'approprie des syllabes constituées de quatre jusqu'à sept lettres en plus de la gestion de leur prononciation: pour le même son, on peut avoir deux émissions de voix différentes en fonction de la consonne à laquelle il est

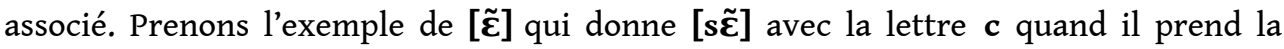
forme in ou la forme ein dans: " ceinture " et "cinquante ». Il est par contre prononcé $[\mathbf{k} \tilde{\varepsilon}]$ quand il prend la forme ain/aim dans « africain » par exemple. Notons 
que les apprenants ont "plus ou moins " pu traiter ces difficultés ce qui a fait que les résultats se sont stabilisés dans un niveau " moyen » et non pas « faible ». Ils se seraient appuyés sur les combinaisons déjà étudiées : ci, ce et ca.

$\mathrm{Au}$ niveau de l'acquisition des sons, une qualité "très faible " dont témoigne un pourcentage de 15,83\%, est enregistrée au cours de cette dernière étape du processus enseignement/apprentissage. Nous avons en effet, commencé la troisième étape par deux séances d'apprentissage durant lesquelles, nous avons proposé des digrammes et des trigrammes c'est-à-dire des graphèmes constitués de deux, voire trois lettres auxquels correspond un seul phonème. Il s'agit des différentes transcriptions des voyelles é, e et $\mathbf{o}$ ainsi que les six voyelles nasales an, en, on, et in. La double difficulté pour l'apprenant résiderait de ce fait, à cette étape, dans la multiplicité graphique. Les différentes transcriptions des voyelles étant au nombre de onze et celle des voyelles nasales au nombre de treize. Les meilleurs scores pour le paramètre "réussite » sont réalisés dans l'ensemble de classes où le nombre d'élèves atteint une moyenne de 21 . Et ce, par rapport aux classes où la moyenne des élèves atteint 24 .

Pour l'expérience professionnelle, les meilleurs résultats concernant ce paramètre sont mentionnés dans les classes des enseignantes qui ont travaillé en moyenne 9 ans; et ce, par rapport aux enseignantes qui ont travaillé en moyenne 3 ans. En ce qui concerne l'emplacement des écoles, les meilleurs scores de réussite sont mentionnés dans toutes les régions concernées par l'étude avec une prédominance des écoles de la région semi urbaine suivie de la région urbaine. Pour le paramètre "déficit", qui comporte rappelons- le, les deux indices : «moyen au niveau de l'acquisition de la combinaison » (MAC) et «très faible dans l'acquisition des sons » (TFAS), les résultats qui témoignent d'un haut niveau de carence tout comme ceux qui témoignent d'un niveau moins important de faiblesse sont indiqués dans les classes où la moyenne des élèves est estimée à 23.

En ce qui est de l'expérience professionnelle des enseignantes, le niveau haut de déficience est signalé chez les enseignantes dont la moyenne des années de service atteint 5 ans. Un niveau de déficience moins important est signalé par contre dans les classes des enseignantes dont la moyenne de l'expérience professionnelle atteint 6 ans de service. L'analyse de l'impact du milieu socio culturel révèle que les écoles des quatre régions concernées par notre étude ont connu un niveau important de déficience (supérieur à la moyenne) dans l'acquisition de l'acte de lire à ce stade de l'apprentissage avec une prédominance dans la région semi urbaine. Un niveau moins important (inférieur à la moyenne) est signalé à égalité dans les régions : semi urbaine, rurale et semi rurale avec une prédominance des écoles de la région urbaine.

Nous récapitulons à travers l'histogramme suivant, la qualité du niveau de rendement au cours de cette deuxième étape de l'enseignement/ apprentissage. 
Fig6 : la qualité de rendement des apprenants durant la troisième étape de l'apprentissage

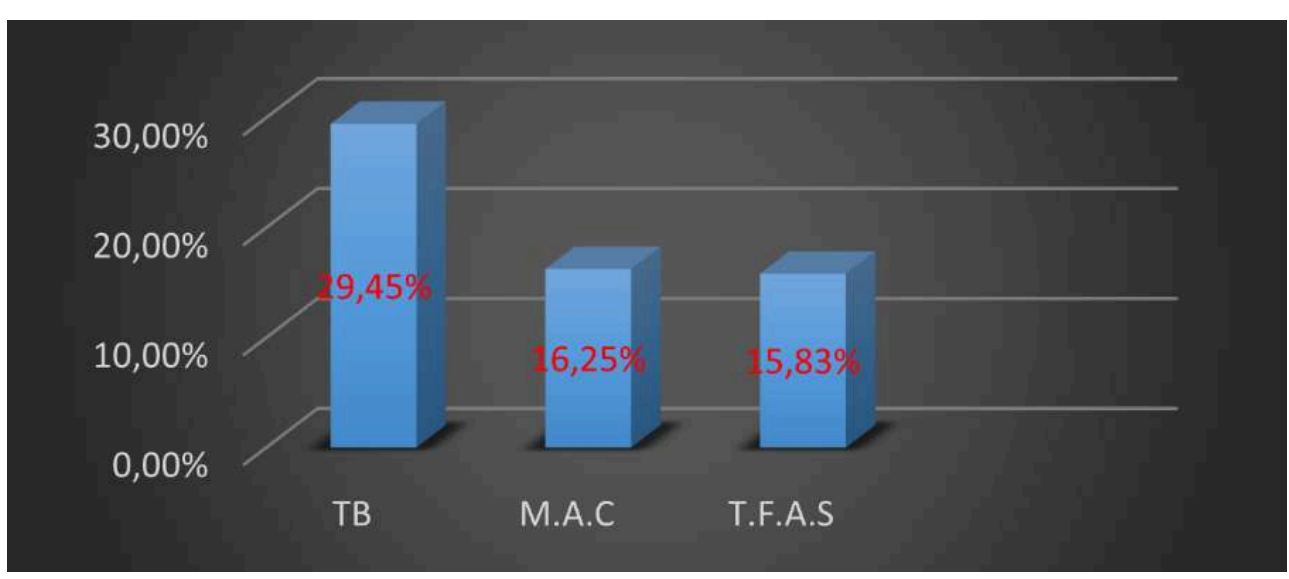

Au terme de cette étude, nous concluons que l'évolution des résultats témoigne d'une rentabilité dès le début de l'apprentissage, de la démarche synthétique. Les premières unités « indispensables " au déchiffrement à savoir lettres, sons ainsi que le principe de combinaison paraissent de ce fait, acquis pour un bon nombre d'apprenants. Nous récapitulons à travers le graphe suivant l'évolution du paramètre « réussite » au cours du parcours enseignement/apprentissage.

Fig7 : évolution de la réussite au cours des trois étapes du processus enseignement/ apprentissage.

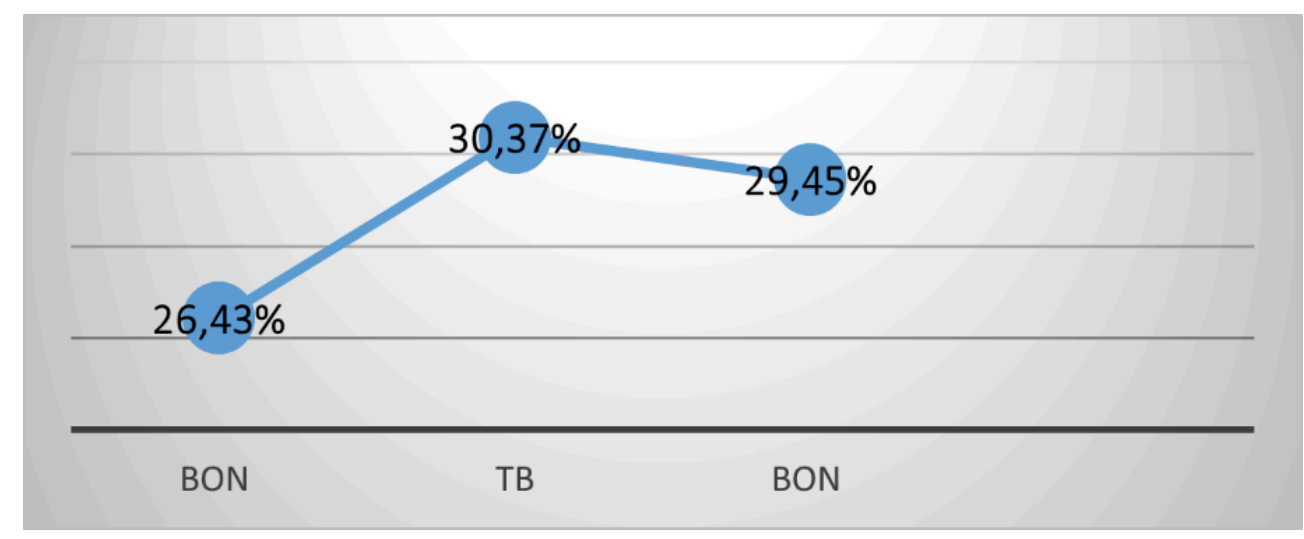

51 En parallèle, un niveau de déficit est signalé tout le long de ce parcours d'apprentissage de la lecture aussi bien au niveau de l'appropriation de la lettre que dans l'appropriation du principe de combinaison. Le paramètre déficit apparait tout le long de l'étude à travers les deux indices «moyen au niveau de l'acquisition de la combinaison " (MAC) et «très faible dans l'acquisition de la lettre/du son » (T.F.A.L/S). Il est à noter que les deux indices ont connu le même parcours d'évolution au cours des trois étapes du processus enseignement/apprentissage. En effet, ils paraissent à travers des scores qui baissent lors de la deuxième étape pour retrouver une certaine hausse vers la troisième.

Encore une fois, le principe «facilitateur" basé sur l'inculcation progressive des éléments impliqués directement dans le décodage parait «efficace » du moment que le déficit connait une régression dans la deuxième étape après que l'apprenant s'habitue à son enseignement et s'entraine à lire; Après quoi, l'insuffisance fléchit quand l'apprenant s'implique dans une étape plus «compliquée " celle de l'acquisition des 
sons complexes avec les difficultés qu'ils présentent aussi bien au niveau de l'identification qu'au niveau de la combinaison. Notons quand même que l'acquisition de la syllabe a été tout le long de ce parcours moyenne mais jamais faible.

Nous récapitulons à présent l'évolution du paramètre " déficit » au cours du parcours enseignement/apprentissage.

Fig7 : évolution du déficit au cours des trois étapes du processus enseignement/apprentissage

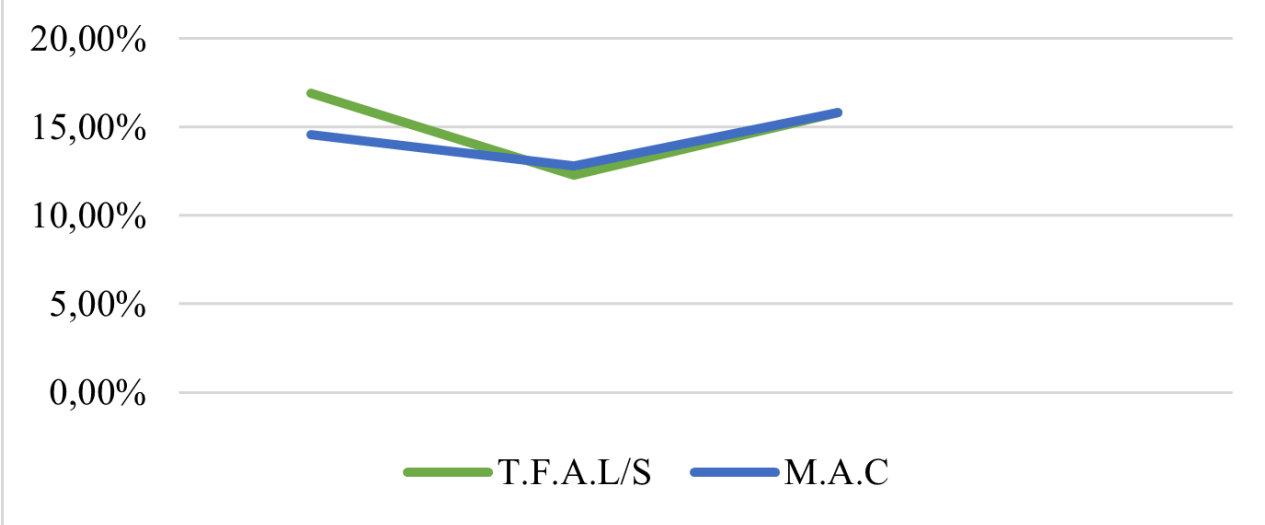

L'aptitude à lire appelle des capacités intellectuelles qui induisent une certaine " invention", des activités langagières et même des connaissances grammaticales et syntaxiques. Malgré la diversité des éléments qui composent l'acte de lire, ce dernier reste en étroite relation avec l'appropriation des graphèmes qui facilitent le décodage ainsi que la compréhension qui constitue la finalité de toute lecture. Apprendre à lire à un enfant à l'école primaire privilégie de ce fait, des pratiques enseignantes " fiables » qui conduiraient l'apprenant au statut du «bon lecteur » surtout que l'appropriation des autres activités dépend de la maitrise de cette activité.

Rappelons que notre étude prend la forme d'une expérimentation menée dans un nombre d'écoles de la wilaya de Constantine et qu'elle tend à prouver à quel point la méthode syllabique basée sur le principe synthétique, serait efficace dans l'inculcation de l'acte de lire à des enfants de troisième année primaire. Nous aspirons à un statut de lecteur autonome en dehors de toute dépendance familiale. Il nous intéresse également de les libérer de la technique de mémorisation des textes condamnés le plus souvent à l'oubli. Les résultats ont prouvé qu'effectivement la méthode syllabique serait adéquate pour enseigner la lecture/ déchiffrement dans nos écoles grâce à ses principes didactiques «facilitateurs » car ils s'organisent autour d'une logique d'apprentissage progressif qui va du plus simple qu'est la lettre vers le plus complexe dont le mot, la phrase et enfin le texte. Les insuffisances décelées n'ont pas eu un impact trop négatif sur ce choix du moment que les indices positifs sont restés prédominants le long de l'évaluation continue.

Malgré cela, nous infirmons relativement le facteur de l'adéquation avec l'effectif quel que soit son importance et le milieu socio culturel de l'enfant avec cette méthode du moment que des classes avec un effectif réduit ont montré les mêmes carences que celles à effectif élevé. Il en est de même pour l'emplacement des écoles où nous avons enregistré la même qualité de rendement positive ou négative aussi bien dans des zones rurales que dans des zones urbaines. Parler de l'efficacité d'une telle ou telle méthode n'exclut en aucun cas l'importance de l'apport de l'enseignant. Bien au contraire, nous 
pensons que la touche du pratiquant à travers des gestes professionnels comme la régulation des insuffisances méthodologiques, l'ajustement de la pratique selon les conditions de l'apprentissage, restent indispensables. Même si l'application de cette méthode dans nos classes s'avère en adéquation avec l'expérience professionnelle des enseignants aussi bien anciens que novices, ceci ne peut exclure la nécessité d'une formation bien ciblée et surtout continue de ces praticiens.

\section{BIBLIOGRAPHIE}

BOULANGER Françoise, A la découverte de la lecture : premiers apprentissages. Pratiques et théories, Ed, sciences humaines 2010.

COURTILLON Janine, Elaborer un cours de FLE, Ed. Hachette, 2003

GIOLLITO Pierre, Histoire de l'enseignement primaire au XIX siècle, Ed. Fernand Nathan, 1984.

Ministère de l'Education nationale, -Document d'accompagnement du programme de français de la 2ème année primaire 2003.

Ministère de l'Education nationale, -Document d'accompagnement du programme de français cycle primaire2016

MUCKENSTURM Pierre, Il faut apprendre à lire comment ? Pourquoi ? Ed. paradigme, Orléans, 2012.

\section{Articles en ligne}

BOCKSTAEL Anne, «Qu'est-ce que lire ? Les pistes théoriques », Revue la filoche [on line], mai, juin, juillet 2007pp 123-132. URL :www.ffedd.be/Cms_files/ffedd/files/filoche/2007_318-13.pdf

CHARMEUX Evelyne, « Mais enfin pourquoi revenir sur l'apprentissage de la lecture ? »,Education, Ecole et Pédagogie[On line],septembre 2016 connection on 27/01/2020URL :charmeux.fr/blog/ index. Php ?2016/09/11/302-mais-enfin-pourquoi- revenirtoujours-sur-l-apprentissage-de-la-lecture

CHAUVEAU Gérard, « Apprendre à lire ou apprendre l'écrit », Revue les cahiers pédagogiques [On line], $\mathrm{n}^{\circ} 479$ connection on 07 mars 2020

URL :https://www.cahiers-pedagogiques.com/Apprendre-a-lire-ou-apprendre-l-ecrit

CHAUVEAU Gérard, Eliane Rogovas Chauveau 1990, « Les processus interactifs dans le savoir lire de base »,Revue française de la pédagogie [On line], 1990 pp 23-30,connection on 23 décembre 2019

URL :ife.ens-lyon.fr > revue-francaise-de-pedagogie > INRP_RF090_3

CUCH Thérèse, Sommer Michelle, « Halte au départ global », collection Léo et Léa [On line], 2016, connection on 28 décembre 2020

URL :www.leolea.org/halte-au-depart-global.par.therese-cuche-et-michell sommer.html

DEHAEN Stanislas, « Les mécanismes cérébraux de la lecture », enregistrement de cours au collège de Franceet au collège de Belgique [On line], 2007, 2011 
URL :https://www.collge-france-.fr/site/stanislas-dehaen/course.2006-2007.htm

GAONAC'H Daniel, « La lecture en langue étrangère : un tour d'horizon d'une problématique de psychologie cognitive » aile revue [online],13|2000, n 13,pp 5-14

URL :aile.revues.org $>$ Numeros $>13$

GIASSON Jocelyne, « La lecture : apprentissage et difficultés » adapté par G. Vandercasteel », graine de lire[Online], novembre2014,connection on 15 décembre 2019

URL :www.grainedelire.fr9wp-content>uploads`sites`2014/11>la lecture.

GOMBERT Jean Emile, « Compétences et processus mobilisés par l'apprentissage de la lecture », Document envoyé au PIREF en vue de la conférence de consensus sur l'enseignement de la lecture à l'école primaire [Online],4-5 décembre 2003 connection on 11 décembre 2019.

URL :http://www.bienlire.education.fr> 2003

PEEREMAN, R., SPRENGER-CHAROLLES, L. \& MESSAOUD-GALUSI, S, « l'apport de la morphologie à la cohérence des relations orthographe-son :uneanalyse quantitative baséesur les lecteurs du primairefrançais ».L'Annéepsychologique[Online]2013113(1),p.3-33.

DOI : $10.4074 /$ S0003503313001012

URL : https://www.cairn.info/revue-l-annee-psychologique1-2013-1-page-3.htm ? contenu $=$ resume

PEIRON Denis, « Apprentissage de la lecture : les méthodes qui marchent »la croix actualité [Online], septembre 2015

URL :www. La-croix.com>actualite>France

RAFONI Jean-Charles, " Apprentissage de la lecture en français langue seconde », organisation des CASNAV[Online], mars 2014

URL :acgrenoble.fr/casnavac/accueil/jcRafoni2014/index.php ?post/2014/06

RIQUOIS Estelle, « Acquérir une compétence lectoriale en français langue étrangère », collection des congrès mondiaux [Online] ,2010,since 12 juillet 2010,connection on 15 février 2020

URL :https://www.linguistiquefrancaise.org/articles/cmlf/abs/2010/01/cmlf2010_000236/ cmlf2010_000236.html

SprengercharollesLilliane, «L'apprentissage de la lecture (du comportement aux corrélats neuronaux), Revuepratiques : linguistique, littérature, didactique [Online],169|170 2016, connection on 01 janvier 2020

URL : https://journals.openedition.org/pratiques/2969

\section{NOTES}

1. 1 Un son constitué de deux lettres comme au- ei...

2. 2 Un son constitué de trois lettres comme eau- œu... 


\section{RÉSUMÉS}

A travers cette étude, nous soulevons la problématique de la répercussion de l'application de la méthode syllabique sur le rendement des apprenants algériens de troisième année primaire quant à l'appropriation de l'habileté de la lecture/déchiffrement. A travers une progression de notre conception, nous menons une expérimentation dans quinze classes de troisième année primaire appartenant à neuf écoles de la circonscription f2 de la wilaya de Constantine. Les résultats nous indiqueront à quel point l'apport de l'application d'une telle démarche serait en faveur d'une bonne maitrise de cette activité. Le questionnaire destiné aux enseignants, permettra d'apprécier l'adéquation des principes méthodologiques de cette méthode avec différentes conditions de travail.

Through this study, we raise the problem of the impact of the syllabic method on the performance of Algerian third-year primary-school learners in terms of reading/deciphering skills. Our research design is based on an experiment conducted with fifteen third-year classes in nine primary schools situated in the f2 district of the wilaya of Constantine. The results will indicate to what extent the contribution of the application of such an approach would be in favor of a good mastery of the reading activity. A questionnaire intended for teachers would allow us to evaluate the adequacy of the methodological principles of this method with in different working conditions.

\section{INDEX}

Mots-clés : Méthode syllabique, enseignement/apprentissage, lecture/déchiffrement, troisième année primaire, français langue étrangère

Keywords : syllabic method, teaching/learning, reading/deciphering, third year of primary school, French as a foreign language

\section{AUTEURS}

\section{AFAF SALHI}

Laboratoire LESMS, faculté des lettres et langues, université de Bejaia

\section{MOURAD BEKTACHE}

Laboratoire LESMS, faculté des lettres et langues, université de Bejaia 